VOL. I (1969), 375-379.

\title{
On boolean near-rings
}

\section{Steve Ligh}

It is well-known that a boolean ring is commutative. In this note we show that a distributively generated boolean near-ring is multiplicatively commutative, and therefore a ring. This is accomplished by using subdirect sum representations of near-rings.

\section{Introduction}

It is well-known that a boolean ring is isomorphic to a subdirect sum of fields $I /(2)$. The purpose of this note is to extend the above result to distributively generated near-rings. Also, examples will be given to show that the result does not hold for arbitrary near-rings.

\section{Definitions and basic information}

A (left) near-ring $R$ is a system with two binary operations, addition and multiplication, such that

(i) the elements of $R$ form a group $(R,+)$ under addition,

(ii) the elements of $R$ form a multiplicative semigroup,

(iii) $x(y+z)=x y+x z$, for all $x, y, z \in R$.

In particular, if $R$ contains a multiplicative semigroup $S$ whose elements generate $(R,+)$ and satisfy

(iv) $(x+y)_{8}=x s+y s$, for all $x, y \in R$ and $s \in S$, we say that $R$ is a distributively generated (d.g.) near-ring.

The most natural example of a near-ring is given by the set $R$ of all mappings of an additive group (not necessarily abelian) into itself. If the mappings are added by adding images and multiolication is iteration,

Received 8 July 1969. 
then the system $(R,+, \cdot)$ is a near-ring. If $S$ is a multiplicative semigroup of endomorphisms of $R$ and $R^{\prime}$ is the sub-near-ring generated by $S$, then $R^{\prime}$ is a d.g. near-ring. Other examples of d.g. near-rings may be found in [6].

An element $a$ of $R$ is right (anti-right) distributive if $(b+c) a=b a+c a((b+c) a=c a+b a)$ for all $b, c \in R$. It follows at once that an element $a$ is right distributive if and only if $(-a)$ is anti-right distributive. In particular, any element of a d.g. near-ring is a finite sum of right and anti-right distributive elements.

The kernels of near-ring homomorphisms are called ideals. Blackett [3] showed that $K$ is an ideal of a near-ring $N$ if and only if $K$ is a normal subgroup of $(N,+)$ that satisfies

(i) $N K \subseteq K$ and

(ii) $(m+k) n-m n \in K$, for all $m, n \in N$ and $k \in K$.

Distributively generated near-rings allow a much stronger structure theory and representation theory than near-rings in general. Many of the fundamental theorems on rings can be generalized to d.g. near-rings. See, for example, [1], [7], [8] and [9].

\section{Subdirect sums of near-rings}

The theory of subdirect sum representation of rings carries over almost word for word to near-rings [5]. A nonzero near-ring $R$ is subdirectly irreducible if and only if the intersection of all the nonzero ideals of $R$ is nonzero. The near-ring analogue of Birkhoff's [2] fundamental result for rings can be stated as follows.

THEOREM 1. [5] Each near-ring $R$ is isomorphic to a subdirect sum of subdirectly irreducible near-rings.

\section{The main result.}

In this section we shall prove that every d.g. boolean near-ring is a ring. To facilitate the discussion we first prove two lemmas.

LEMMA 1. Let $R$ be a d.g. boolean near-ring and let $x, y, z, w$ be elements in $R$ such that $x$ and $y$ are right distributive, $z$ is anti-right distributive and $w$ is any element in $R$. Then the following 
statements hold.

$$
\begin{aligned}
& \text { (i) } x+x=0, \\
& \text { (ii) } x y=y x, \\
& \text { (iii) } x z=z x, \\
& \text { (iv) } x w=w x, \\
& \text { (v) } A_{x}=\{r \in R: x r=0\} \text { is an ideal of } R, \\
& \text { (vi) If } A_{x}=0 \text {, then } x \text { is an identity and }(R,+) \text { is abelian. }
\end{aligned}
$$

Proof. (i) follows from the expansion of $(x+x)^{2}$. Since $x y$ is right distributive, $(i i)$ is the consequence of $(i)$ and the expansion of $(x+y)^{2}$. For $(i i i)$, observe that $(-z)$ is right distributive and hence $x(-z)=(-z) x$. Since $x$ is right distributive $(-z) x=-(z x)$. It follows that $x z=z x$ because $x(-z)=-(x z)$ is always valid. Since every element of a d.g. near-ring is a finite sum of right and anti-right distributive elements, we have, by using (ii) and (iii), that

$$
\begin{aligned}
x w=x\left(w_{1}+w_{2}+\ldots+w_{n}\right) & =x w_{1}+x w_{2}+\ldots+x w_{n} \\
& =w_{1} x+w_{2} x+\ldots+w_{n} x \\
& =w x .
\end{aligned}
$$

The proof of $(v)$ follows from $(i v)$ and the definition of an ideal. If $A_{x}=0$, then $x$ is a left identity. For if not, there exists $y \in R$ such that $y \neq 0$ and $x y \neq y$. Thus $x(x y-y)=0$ and, $A_{x} \neq 0$, which is a contradiction. By ( $i v)$ and $(i), x$ is a two-sided identity and $x+x=0$. If $r$ is any element of $R$ then $r+r=r(x+x)=0$. Thus each element of $(R,+)$ is of order two and $(R,+)$ is abelian.

LEMMA 2. If $R$ is a subdirectly irreducible d.g. boolean near-ring then $R$ is a boolean ring with an identity.

Proof. Suppose for each right distributive element $x$ in $R, A_{x} \neq 0$. Since $R$ is subdirectly irreducible and each $A_{x}$ is an ideal of $R$, we have that $\cap^{A} x=A \neq 0$. Let $w \neq 0$ be an element in $A$. Thus $x w=0$ for each right distributive element $x$ in $R$. Since $x w=w x=0$, it follows that $w z=0$ if $z$ is anti-right distributive. Furthermore, if 
$y$ is any element in $R$, then $w y=w\left(y_{1}+y_{2}+\ldots+y_{n}\right)=w y_{1}+w y_{2}+\ldots+w y_{n}=0$. This implies that $A_{w}=R$. But then $w=w w=0$, contradicting the fact that $w \neq 0$. Thus there exists a right distributive element $x$ in $R$ such that $A_{x}=0$. By (vi) of Lemma $l,(R,+)$ is abelian and $x$ is an identity. Now that $R$ is a ring follows from $[6, \mathrm{p} .93]$.

We are now ready to prove the main result of this note.

THEOREM 2. Every d.g. boolean near-ring $R$ is a boolean ring.

Proof. By Theorem l, $R$ is isomorphic to a subdirect sum of subdirectly irreducible near-rings $R_{i}$. Now each $R_{i}$ is a homomorphic image of $R$ and therefore a d.g. boolean near-ring [6]. By Lemma 2, each $R_{i}$ is a boolean ring and hence $\left(R_{i},+\right)$ is abelian. It follows that $(R,+)$ is abelian and hence $[6, \mathrm{p} .93] R$ is a ring.

\section{General boolean near-rings}

Let $G$ be an additive group (not necessarily abelian). Define for each $x \in G, x y=y$ for each $y \in G$. Then $(G,+, \cdot)$ is a boolean near-ring. Other interesting examples of boolean near-rings which are not rings can be found in [4] and [11]. Clearly there exist boolean near-rings which are not boolean rings. Thus we conclude that Theorem 2 cannot be extended to arbitrary near-rings.

\section{Remark}

A ring $R$ is said to be a p-ring if $p$ is a fixed prime and ${ }^{p}=x, p x=0$ for each $x$ in $R$. Thus a boolean ring is a 2-ring. McCoy and Montgomery [10] showed that a p-ring $R$ is isomorphic to a subdirect sum of fields $I /(p)$. In the light of the result of this paper one naturally asks that whether the result of McCoy and Montgomery can be extended to distributively generated near-rings. This question is still open. 


\section{References}

[1] James C. Beidleman, "Distributively generated near-rings with descending chain condition", Math. z. 91 (1966), 65-69.

[2] Garrett Birkhoff, "Subdirect unions in universal algebra", Bull. Amer. Math. Soc. 50 (1944), 764-768.

[3] D.W. Blackett, "Simple and semisimple near-rings", Proc. Amer. Math. Soc. 4 (1953), 772-785.

[4] James R. Clay and Donald A. Lawver, "Boolean near-rings", Canad. Math. Buzz. (to appear).

[5] Charles Gilbert Fain, "Some structure theorems for near-rings", Doctoral thesis, University of Oklahoma, 1968.

[6] A. Fröhlich, "Distributively generated near-rings, (I. Ideal Theory)", Proc. London Math. Soc. (3) 8 (1958), 76-94.

[7] Steve Ligh, "On distributively generated near-rings", Proc. Edinburgh Math. Soc. (to appear).

[8] Steve Ligh, "On division near-rings", Canad. J. Math. (to appear).

[9] Steve Ligh, "Near-rings with descending chain condition", Compositio Math. (to appear).

[10] N.H. McCoy and Deane Montgomery, "A representation of generalized boolean rings", Duke Math. J. 3 (1937), 455-459.

[11] N.V. Subrahmanyam, "Boolean semirings", Math. Ann. 148 (1962), 395-401.

University of Florida,

Gainesville, Florida. 